Proceedings

\title{
Design and Optimization of a Low-Resonant- Frequency Piezoelectric MEMS Energy Harvester Based on Artificial Intelligence ${ }^{\dagger}$
}

\author{
Seyedfakhreddin Nabavi * and Lihong Zhang \\ Department of Electrical and Computer Engineering, Faculty of Engineering and Applied Science, \\ Memorial University of Newfoundland, St. John’s, NL A1B 3X9, Canada; lzhang@mun.ca \\ * Correspondence: snabavi@mun.ca; Tel.: +1-709-743-2744 \\ † Presented at the Eurosensors 2018 Conference, Graz, Austria, 9-12 September 2018.
}

Published: 26 November 2018

\begin{abstract}
In this study we propose a piezoelectric MEMS vibration energy harvester with the capability of oscillating at low (i.e., less than $200 \mathrm{~Hz}$ ) resonant frequency. The mechanical structure of the proposed harvester is comprised of a doubly clamped cantilever with a serpentine pattern associated with several discrete masses. In order to obtain the optimal physical aspects of the harvester and speed up the design process, we have utilized a deep neural network, as an artificial intelligence (AI) method. Firstly, the deep neural network was trained with 108 data samples gained from finite element modeling (FEM). Then this trained network was integrated with the genetic algorithm (GA) to optimize geometry of the harvester to enhance its performance in terms of resonant frequency and generated voltage. Our numerical results confirm that the accuracy of the network in prediction is above $90 \%$. Consequently, by taking advantage of this efficient AI-based performance estimator, the GA is able to reduce the device operational frequency from $169 \mathrm{~Hz}$ to $110.5 \mathrm{~Hz}$ and increase its efficiency on harvested voltage from $2.5 \mathrm{~V}$ to $3.4 \mathrm{~V}$ under $0.25 \mathrm{~g}$ excitation.
\end{abstract}

Keywords: low-resonant-frequency; MEMS harvester; piezoelectric; deep neural networks; artificial intelligence; genetic algorithm; design automation

\section{Introduction}

To provide a sustainable power supply for the low-power electronic applications, the vibration energy harvesting technique, which converts kinetic energy of ambient vibration resources such as wind flow [1], human motion [2], etc. to electricity, has been recognized as a feasible solution. In this regard, the ambient vibration can be converted to electricity by electrostatic-, electromagnetic- or piezoelectric-based techniques. Each technique has its own advantages as well as drawbacks. At the micrometer scale, the piezoelectric-based technique has gained a lot of interests due to the compatibility of piezoelectric martial deposition with the regular micromachining processes [3] and their high electromechanical conversion efficiency [4].

The silicon-based piezoelectric MEMS energy harvesters normally have a high operational resonate frequency (i.e., in the range of $500 \mathrm{~Hz} \sim 10 \mathrm{kHz}$ ) owning to their small physical aspects and the utilization of the silicon wafer as a substrate, which is strongly stiff and brittle. In contrast, the natural frequencies of the ambient vibration resources are mostly less than $200 \mathrm{~Hz}$ [5]. Thus, there has been multiple endeavors in the literature to offer the piezoelectric MEMS energy harvesters with the capability of operating in the real environment. Using a big proof mass at the tip of the piezoelectric cantilever was proposed as a practical method for resonant frequency reduction and conversion efficiency enhancement of the MEMS harvesters. In our previous study [6], the 
multimodal behavior, which was obtained by using three discrete proof masses at different locations within the MEMS harvester, could successfully reduce the device resonant frequency. In more recent studies, the capability of micro-fabricated spiral [7] and T shaped [8] piezoelectric energy harvesters were investigated. It was shown that such energy harvesters were more suitable than the conventional ones for being utilized in the real environment.

As a matter of fact, the obtained frequency reduction in the prior studies is not that considerable, which means further reduction along with the energy conversion efficiency enhancement for the silicon-based piezoelectric harvesters to make them a better fit for the operation in the real environment is highly demanded. Therefore, in this study a doubly clamped cantilever with a serpentine pattern associated with several proof masses is proposed for resonant frequency reduction and efficiency improvement. Our proposed structure can offer sound features in reducing the MEMS geometry stiffness and increasing the total weight. Moreover, to optimize the performance of the proposed MEMS energy harvester, a new optimization methodology based on Artificial Intelligence (AI) has been also proposed. In this regard, a deep neural network for prediction of resonant frequency and harvested voltage is trained. Thereafter, this network is integrated with a geneticalgorithm-based optimization platform for tuning the harvester dimensions

\section{Low-Resonant-Frequency Piezoelectric MEMS Harvester}

The 3-D structural diagram of the proposed low-resonant-frequency MEMS vibration is illustrated in Figure 1. This energy harvester comprises seven piezoelectric cantilevers, which are connected together by the proof masses. Thus, the overall device can be overviewed as a serpentine cantilever, which is clamped on two sides. In comparison to the conventional clamped-free piezoelectric cantilevers, the serpentine cantilever can increase the device deflection. Moreover, by using seven discrete proof masses, we can provide the uniform mass distribution along the harvester geometry. Consequently, with this structure lower mechanical stiffness and larger overall mass are attained. In the following sections, the capability of the proposed structure in harnessing the vibration energy will be discussed.

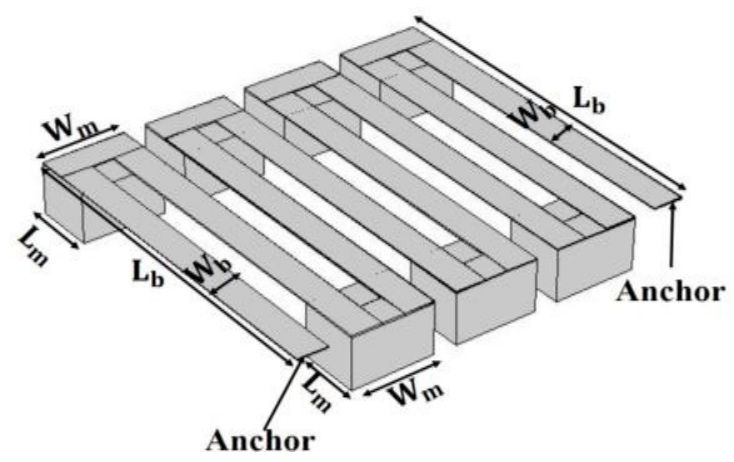

Figure 1. Structural diagram of the proposed low-resonant-frequency piezoelectric MEMS energy harvester.

\section{AI-Based Optimization Methodology}

In order to obtain the optimum physical aspects of the proposed piezoelectric MEMS harvester in terms of resonant frequency and harvested voltage, the AI- based optimization method, where a deep neural network (DNN) is integrated with genetic algorithm (GA) has been developed. The working principle of the DNN on the decision making process is inspired by human brains. It is categorized as an artificial intelligence technique. In this study, the displayed DNN in Figure 2 was implemented in MATLAB to minimize computation time of the optimization process. As shown in this figure, the utilized network consists of three different layers, namely, input, hidden and output layers, respectively. In the input layer four optimizable variables, i.e., $L b, w_{b}, L m, w_{m}$, as indicated in Figure 1, can be imported to the network. The hidden layer, which is comprised of seven individual layers, is sequentially made up of sigmoid neurons of $60,55,45,30,20,10$ and 5, respectively. 
Eventually, the output layer for reporting the amounts of both resonant frequency and harvested voltage is formed with two neurons. At the first step, the constructed DNN was trained with 108 data sets, which were obtained by conducting the FEM simulations with COMSOL Multiphysics software.

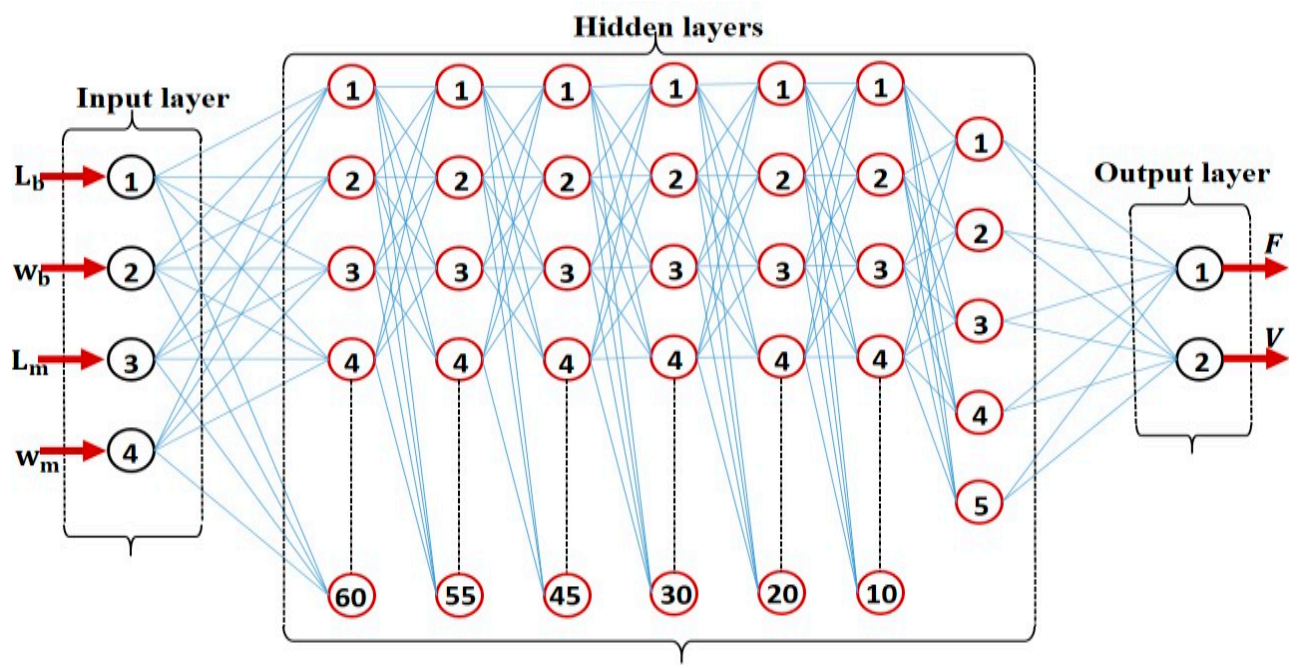

Figure 2. Illustration of the utilized deep neural network (DNN) for estimating the performance of the proposed low-resonant-frequency piezoelectric MEMS energy harvester.

Once the network was trained and its accuracy level was confirmed, the GA, which is an evolutionary computation method for optimizing complex problems [9], was integrated with the DNN. Therefore, the algorithm generates the random numbers for the input variables and their effects on the performance of the energy harvester in terms of resonant frequency and harvested voltage were evaluated within a few seconds. Finally, the GA selects those variables, which can provide both lower resonant frequency and higher amplitude of harvested voltage, as the optimal solutions.

\section{Results and Discussion}

To demonstrate the effectiveness of the proposed AI-based optimization methodology, we defined an un-optimized structure, where the optimizable variables (i.e., $L b, w_{b}, L_{m}$ and $w_{m}$ ) were chosen to be the median values as per their allowed ranges. To improve the performance out of the un-optimized devices, our AI-based optimization method was executed with the population size of 20 and the generation number of 50 for the GA setup. Further information regarding the selection of these two user-defined factors can be found in [10]. The obtained results for the un-optimized (unopt) and optimized (opt) MEMS energy harvesters are listed in Table 1. In this table, the allowed ranges for the optimizable parameters, which are specified by their design rule constraints, are listed in the second rows. The computed resonant frequencies by the DNN and FEM simulations for the un-optimized and optimized structures are presented in the 6th and 7th columns, while the estimated harvested voltages by the DNN and FEM simulations are listed in the 8th and 9th columns, respectively.

Table 1. Performance evaluation of the un-optimized and optimized proposed low-resonantfrequency piezoelectric MEMS harvester by using the DNN and FEM simulations.

\begin{tabular}{ccccccccc}
\hline Parameter & $L b[\mu \mathrm{m}]$ & $w_{b}[\mu \mathrm{m}]$ & $L_{m}[\mu \mathrm{m}]$ & $w_{m}[\mu \mathrm{m}]$ & $\begin{array}{c}\text { Freq. } \\
(\mathrm{DNN}) \\
{[\mathrm{Hz}]}\end{array}$ & $\begin{array}{c}\text { Freq. } \\
(\text { FEM) } \\
{[\mathrm{HZ}]}\end{array}$ & $\begin{array}{c}\text { Voltage } \\
\text { (DNN) } \\
{[\mathrm{V}]}\end{array}$ & $\begin{array}{c}\text { Voltage } \\
\text { (FEM) } \\
\text { [V] }\end{array}$ \\
\hline Range & $(1,550,3050)$ & $(150,250)$ & $(500,1000)$ & $(600,614)$ & - & - & - & - \\
un-opt & 2300 & 200 & 750 & 607 & 162 & 169 & 2.7 & 2.5 \\
opt & 2945 & 216 & 907 & 609 & 105 & 110.5 & 3.5 & 3.25 \\
\hline
\end{tabular}


From the summarized data in Table 1, it can be seen that the DNN can estimate both resonant frequency and harvested voltage with over $90 \%$ accuracy in reference to the FEM simulations. Therefore, by utilizing the DNN as a performance simulator, we can significantly reduce the required computation time dedicated to the FEM simulations. On the other hand, by this computation time reduction, a larger number of optimizable variables can be testified. Hence, the effectiveness of the optimization algorithm in entrapping the global optima can be further enhanced.

Frequency spectra of the un-optimized and optimized piezoelectric MEMS energy harvesters, which were computed by the FEM simulations under the excitation of $0.25 \mathrm{~g}$, are illustrated in Figure 3. It is shown that the un-optimized device operates at $169 \mathrm{~Hz}$, while it can generate the maximum voltage of $2.5 \mathrm{~V}$. In contrast, by optimizing the harvester dimensions, its resonant frequency can be reduced to $110.5 \mathrm{~Hz}$, and its capability of the voltage harvesting can be considerably enlarged to $3.4 \mathrm{~V}$.

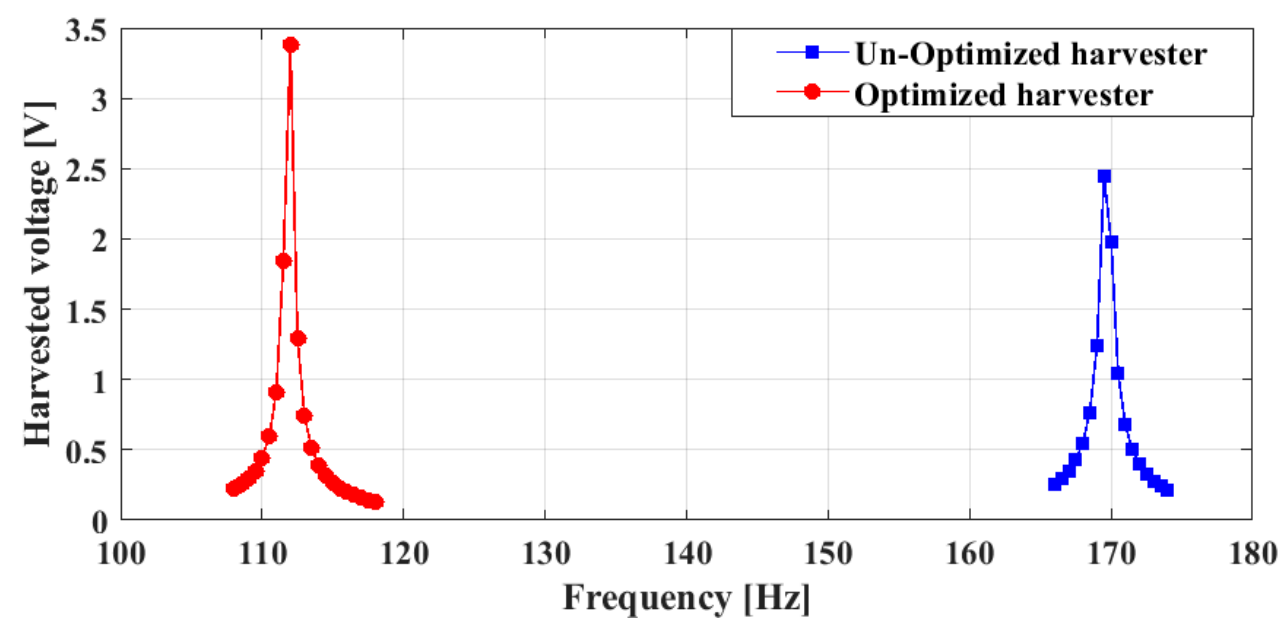

Figure 3. Frequency spectra of the un-optimized and optimized low-resonant-frequency piezoelectric MEMS energy harvesters.

\section{Conclusions}

In this paper, we proposed a low-resonant-frequency piezoelectric MEMS energy harvester. In order to optimize its performance, an AI optimization methodology based on DNN and GA was proposed. It is shown that the trained network could estimate the device performance with accuracy of over $90 \%$. By taking advantage of this efficient AI-based performance estimator, the GA was able to reduce the device operational frequency from $169 \mathrm{~Hz}$ to $110.5 \mathrm{~Hz}$ and increase its energy conversion efficiency in terms of harvested voltage from $2.5 \mathrm{~V}$ to $3.4 \mathrm{~V}$ under $0.25 \mathrm{~g}$ excitation.

Acknowledgments: This work was supported in part by the Natural Sciences and Engineering Research Council of Canada (NSERC), Canada Foundation for Innovation (CFI), Research and Development Corporation (RDC) of Newfoundland and Labrador (through its Industrial Research and Innovation Fund and ArcticTECH R\&D Award), and Memorial University of Newfoundland.

Conflicts of Interest: The authors declare no conflict of interest.

\section{References}

1. Nabavi, S.; Zhang, L. Portable wind energy harvesters for low-power applications: A survey. Sensors 2016, 16, 1101.

2. Kim, M.-O.; Pyo, S.; Oh, Y.; Kang, Y.; Cho, K.-H.; Choi, J.; Kim, J. Flexible and multi-directional piezoelectric energy harvester for self-powered human motion sensor. Smart Mater. Struct. 2018, 27, 035001.

3. Wang, Q.; Lu, Y.; Mishin, S.; Oshmyansky, Y.; Horsley, D.A. Design, fabrication, and characterization of scandium aluminum nitride-based piezoelectric micromachined ultrasonic transducers. J. Microelectromech. Syst. 2017, 26, 1132-1139.

4. Roundy, S.; Wright, P.K. A piezoelectric vibration based generator for wireless electronics. Smart Mater. Struct. 2004, 13, 1131. 
5. Roundy, S.; Wright, P.K.; Rabaey, J. A study of low level vibrations as a power source for wireless sensor nodes. Comput. Commun. 2003, 26, 1131-1144.

6. Nabavi, S.; Zhang, L. Design and optimization of wideband multimode piezoelectric MEMS vibration energy harvesters. Proceedings 2017, 1, 586.

7. Song, H.-C.; Kumar, P.; Maurya, D.; Kang, M.-G.; Reynolds, W.T.; Jeong, D.-Y.; Kang, C.-Y.; Priya, S. UltraLow Resonant Piezoelectric MEMS Energy Harvester With High Power Density. J. Microelectromech. Syst. 2017, 26, 1226-1234.

8. Nabavi, S.; Zhang, L. Design and optimization of MEMS piezoelectric energy harvesters for improved efficiency. In Proceedings of the IEEE SENSORS, Glasgow, UK, 29 October-1 November 2017; pp. 1-3.

9. Nabavi, S.; Zhang, L. MEMS piezoelectric energy harvester design and optimization based on Genetic Algorithm. In Proceedings of the IEEE International Ultrasonics Symposium (IUS), Tours, France, 18-21 September 2016; pp. 1-4.

10. Nabavi, S.; Zhang, L. Design and Optimization of Piezoelectric MEMS Vibration Energy Harvesters Based on Genetic Algorithm. IEEE Sens. J. 2017, 17, 7372-7382.

(C) 2018 by the authors. Licensee MDPI, Basel, Switzerland. This article is an open access article distributed under the terms and conditions of the Creative Commons Attribution (CC BY) license (http://creativecommons.org/licenses/by/4.0/). 